\title{
Epidemiology and Culture
}

\author{
James A. Trostle
}

Resenha - DOI: 10.3395/reciis.v2i1.164pt

\section{Marina D. Cardoso}

Programa de Pós-Graduação em Antropologia, Universidade Federal de São Carlos, São Carlos, Brasil mdcardoso@uol.com.br

O livro de James A. Trostle trata das relações entre epidemiologia e antropologia, mais particularmente a área ou sub-área da antropologia que veio a ser conhecida como "antropologia médica". Mesmo que possam pesar considerações acerca dessa denominação como uma área da antropologia social aplicada à saúde e seus determinantes de ordem cultural, a "antropologia médica" é uma área que vem progressivamente sendo reconhecida nos Estados Unidos e na Europa. Parte desse reconhecimento e da sua progressiva consolidação como área de pesquisa acadêmica, particularmente no âmbito das ciências humanas em saúde, deve-se às questões que estão sendo abordadas neste livro, que urge por uma colaboração mais intensiva entre epidemiologia e antropologia no próprio campo da formulação e intervenção de políticas de saúde pública destinadas a prevenir fatores de riscos de doenças epidemiologicamente identificados.

Talvez o principal argumento que sustenta essa concepção e é ilustrativo da problemática tratada no livro tenha sido a constatação do autor do prefácio dessa edição, S. Leonard Syme, professor de epidemiologia da Universidade da Califórnia (Berkeley): “(...) people have been informed about the things they need to do (to avoid disease risks), and they have failed to follow our advice" (p. xi). Sob essa perspectiva, o autor do livro procura introduzir noções básicas sobre o modo como as concepções sobre doença e saúde, os fatores etiológicos e a própria intervenção terapêutica são amplamente determinados pela "cultura" de um determinado grupo ou comunidade, que também responde sobre a sua diversidade. O termo "cultura”, aqui, não é explicitamente definido pelo autor que, em uma nota de pé de página refere-se tanto às possíveis

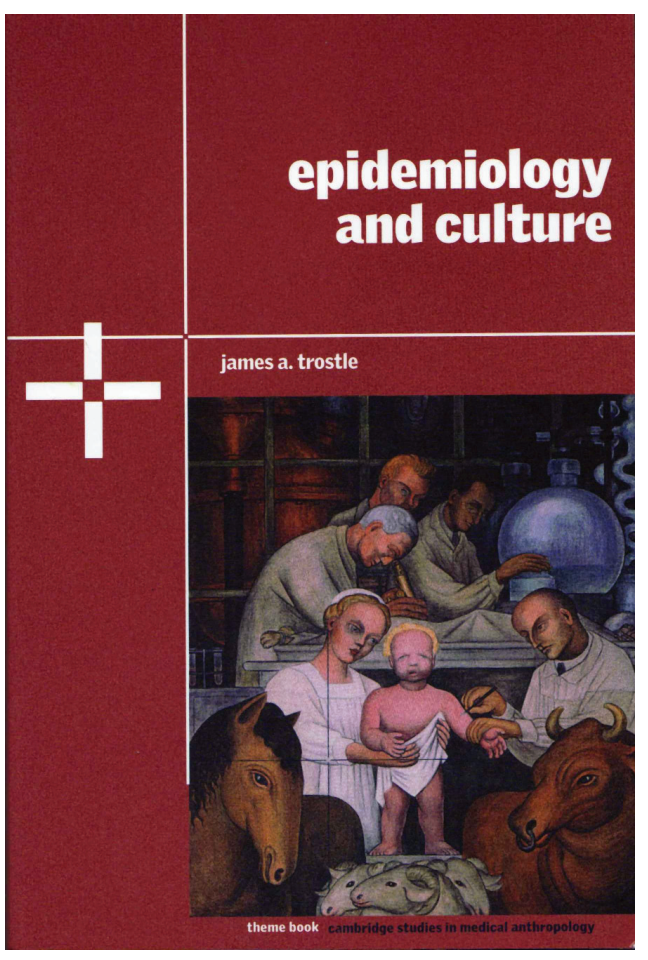

Cambridge, Cambridge
University Press, 2005

ISBN: 978-052179389-6 
definições do conceito como "modo de vida de um povo" até a formulação de Geertz sobre cultura como "conjunto de símbolos que são organizados em sistemas de significação" (p. 5). Mas, à aparente falta de imprecisão do conceito pelo autor, subjaz uma concepção amplamente difundida em todo o texto do livro de cultura como “padrões de comportamento”, o que não só é compatível com a influência da chamada "escola norte-americana" no campo do pensamento antropológico, mas até o seu ulterior emprego para simultaneamente identificar estes padrões com os "padrões de riscos" que os estudos de caráter epidemiológico procurariam revelar.

O livro está organizado de modo a introduzir o leitor, especialmente profissionais da saúde e epidemiologistas, na perspectiva que atribuie importância à "cultura" como fator a ser considerado nos estudos epidemiológicos. De fato, a própria epidemiologia como ciência seria culturalmente "orientada", porque embasada em concepções sobre risco, e, mais particularmente, sobre noções e correlações entre "pessoa", "tempo" e "lugar" que seriam próprias a "cultura" científica ocidental. O capítulo 1 introduz o leitor a essas definições mais genéricas sobre epidemiologia e antropologia médica, assim como já dispõe o argumento central que perpassa todo o livro, que é sobre a necessidade de estudos que integrem uma abordagem interdisciplinar entre epidemiologia e antropologia, designada como "epidemiologia cultural", e que focaria os efeitos do comportamento e das crenças sobre questões relacionadas à saúde. O capítulo 2 procura reconstituir historicamente as origens desta perspectiva de uma abordagem integrada entre essas duas áreas, analisando o modo como fatores sociais e culturais foram incorporados ao campo das investigações e intervenções de cunho epidemiológico.

Tanto estes capítulos como os seis capítulos subseqüentes, pelos quais o livro está organizado, são permeados por exemplos ilustrativos de pesquisas e intervenções na área de saúde pública que usaram esta abordagem interdisciplinar, assim como indicações de leituras suplementares. Os capítulos 3 e 4 procuram, mais explicitamente, ressaltar as dimensões culturais das variáveis pessoa (de acordo com gênero, idade, status marital, ocupacional, sócio-econômico, étnico e religioso), lugar (limites territoriais e políticos) e tempo (escala de referência temporal dos dados coletados), usadas na pesquisa epidemiológica, bem como das próprias condições da coleta de dados (entendida, ela própria, como processo de "troca social") e das noções de medida, probabilidade e risco que fazem parte do escopo conceitual da constituição da epidemiologia como disciplina. Sob esse aspecto, o argumento central do livro é que a própria epidemiologia é uma prática cultural, no sentido que as variáveis e medidas com as quais opera, ou ainda, a forma como as informações advindas das análises epidemiológicas são disseminadas e orientam a formulação de políticas em saúde, são também culturalmente orientadas. O autor observa que uma "epidemiologia cultural" procuraria justamente "(...) reveals the ways in which measurement, causal thinking, and intervention design are all influenced by belief and habit in addition to deduction and rational decision-making" (p.173).
O capítulo 5 ilustra mais especificamente essa argumentação central do livro, ou seja, a necessidade de uma pesquisa quantitativa e analiticamente integrada entre epidemiologia e antropologia, a partir da cólera, conquanto o capítulo 6 foca mais explicitamente sobre a contribuição dessa integração sob o ângulo da formulação de políticas públicas dirigidas a grupos populacionais específicos, quer sob o ângulo do "compartilhamento cultural" de acepções sobre saúde, cuidados e doenças, quer sob o ângulo de padrões diferenciados de perfis epidemiológicos. Este capítulo é o que mais notadamente reflete sobre as contribuições entre as duas áreas naquilo que concerne à formulação e intervenção de políticas de saúde sob o ângulo da sua "eficácia", ou "efetividade".

Ressaltando que as intervenções em saúde são ou deveriam ser formas de comunicação de dados e informações de cunho epidemiológico a serem compartilhados, o autor aponta para a necessidade da participação da comunidade na formulação e gestão das políticas em saúde para a sua maior efetividade. Também conclama os antropólogos a se inserirem de maneira mais efetiva na formulação dessas políticas, por meio da concepção de uma "antropologia participativa", ou seja, o uso dos dados das pesquisas antropológicas como recurso para a informação, mediação, e comunicação entre os gestores em saúde, epidemiologistas e a comunidade, assim como a participação efetiva do antropólogo na intervenção pretendida. O capítulo seguinte, o sétimo, reflete o conjunto dessas proposições articulado a partir de uma noção central à epidemiologia que é o conceito de "risco", apontando quer para as variações das representações e percepções populares e profissionais sobre essa noção, quer para os modos da sua comunicação. Por enfim, o último capítulo apresenta a título de conclusão um sumário das principais questões desenvolvidas ao longo do livro e, retomando a sua argumentação principal, defende a interdisciplinaridade como o caminho profícuo para o balizamento dos dados epidemiológicos, particularmente no que concerne ao seu papel como informação que orienta a formulação e a implementação das políticas em saúde.

Trata-se de um livro essencialmente didático, tanto para epidemiologistas quanto para antropólogos, ou demais interessados nessas abordagens disciplinares e potencialmente interdisciplinares em saúde. Os conceitos usados procuram ser expostos de maneira acessível a qualquer leitor, assim como a exposição do tema recorre sempre a exemplos de pesquisas já realizadas, procurando mostrar quão profícua elas foram para sustentar, inclusive, o argumento central do autor do livro sobre a necessidade do balizamento dos dados epidemiológicos pela "cultura", sobrepondo à "epidemiologia social" uma "epidemiologia cultural", ou seja, balizada pelas representações ou categorias de cunho "popular" sobre doença, cura, e cuidados em saúde. O autor também procura articular uma ampla revisão bibliográfica sobre os temas a que se propõe a analisar, recorrendo a pesquisas realizadas em vários e distintos países e apresentando dados epidemiológicos referentes a diversos agravos (cólera, malária, epilepsia etc.), índices de mortalidade ou de prevalência de doenças de acordo com o tipo de 
serviço de atenção à saúde, dentre outros, para dar suporte ao argumento central do livro sobre a necessidade desses dados serem analisados à luz de determinantes de ordem cultural.

O caráter didático do livro é, nesse sentido, também amplificado pela demonstração empiricamente datada da possibilidade desse tipo de pesquisa "culturalmente orientada" vir a ocorrer no âmbito da epidemiologia pelo uso recorrente de exemplos de pesquisas conduzidas sob esta ótica, ou de dados estatísticos que apontam para a variabilidade dos próprios dados epidemiológicos de acordo com o contexto da sua coleta e mesmo do modo como foi formulado o conjunto de questões e as variáveis que compõem o modelo de análise e inferência epidemiológica. Dado esse caráter "demonstrativo" e didático do texto, difere de outros livros que ou apresentam resultados originais de pesquisas conduzidas sob esse enfoque, ou procuram formular um modelo de análise que integre conceitualmente e metodologicamente a análise antropológica ao campo das pesquisas em saúde (tal como Kleinman, por exemplo ${ }^{1}$ ). Trata-se, assim, muito mais de um livro que procura argumentar, por meio da menção a dados de pesquisas, sobre a necessidade e a importância da interdisciplinaridade com a antropologia médica para a área da epidemiologia, do que propriamente formular um modelo metodológico de referência para esse tipo de pesquisa interdisciplinar.

Deve, no entanto, ser observado que o livro baseiase fundamentalmente em conceitos, postulados e referências bibliográficas de uma corrente da antropologia e antropologia médica norte-americana, ou a autores que as têm como referência. Sob esse aspecto, à clareza da exposição dos motivos pelos quais as pesquisas epidemiológicas deveriam levar em conta os contextos culturais do adoecimento e dos cuidados em saúde, por exemplo, ao sobrepor "padrões culturais" a "padrões epidemiológicos" revela uma tendência do autor a ver no comportamento e nas representações sobre doença e cuidados o fator primordial para a incidência de agravos e índices epidemiológicos que podem resultar, eventualmente, em políticas preventivas em saúde orientadas por ações pedagógicas e re-educadoras para "reforçar a mudança do comportamento" de determinados grupos populacionais (p. 133), o que tende a entrar em conflito com o código simbólico mais geral de referência que sustentam estas representações.

Tem sido a tendência mais geral desta corrente, para dar conta dos impasses decorrentes do confronto entre concepções distintas do adoecimento e dos cuidados em saúde (poder-se-ia dizer, uma mais "técnico-operacional", representados pelos gestores e/ou profissionais da saúde, e outra mais "popular"), glosar as concepções de cunho "popular" mediante um procedimento analítico que as remetem às diferenciações no modo de formular o modelo etiológico e terapêutico (quando não a hábitos e crenças fragmentados e susceptíveis de serem alterados por meio da difusão de conhecimentos médicos, e mesmo intervenção), quando, na verdade, trata-se de modelos radicalmente distintos, articulados por lógicas também distintas. Mesmo que esse fato não impeça que possam vir a ocorrer interpenetrações entre modelos, particularmente, no caso de alguns procedimentos terapêuticos tal como o uso de medicamentos, é a diferença que deve ser radicalizada como produtora efetivamente de um outro modelo, de um outro discurso e, portanto, de outra práxis.

Mais do que isto, entretanto, a urgência para que membros da comunidade participem da elaboração das políticas públicas é que eles possam igualmente identificar quais são, para eles, os problemas mais prementes de atenção à saúde, muitas vezes diretamente relacionados às condições mais gerais de vida, e das quais os problemas referentes ao adoecimento e aos cuidados de atenção à saúde encontram-se diretamente relacionados, mas por lógicas distintas. Tal como o prefaciador do livro comenta: "We in public health have important messages to give to people, but people have lives to lead" (p. xii). O próprio autor defende, a partir de argumentos elaborados por antropólogos, que : “ (...) planned change in communities must begin with extensive community consultations, emerge from local definitions of need, and be continuously subject to local review and adjustment over time" (p.140). O que deve ser observado é que não se trata simplesmente de um processo de "consulta" (consultative process), mas como pode-se analisar as intervenções em saúde hoje no Brasil, tal como elas são implementadas pelos agenciadores de políticas de saúde e os profissionais da área, trata-se, substancialmente, de promover a integração dos grupos populacionais ou "comunidades" a quem elas são dirigidas como efetivos gestores, promotores e planejadores dessas políticas, justamente por operarem em códigos distintos do reconhecimento das situações de risco e do modo de preveni-las.

O livro, em suma, é oportuno para a reflexão não só dos epidemiologistas e antropólogos, mas também para os demais agenciadores e profissionais da área da saúde, pela constatação de que não se tem adequado de forma efetiva a formulação e a operacionalização das políticas públicas para atender às novas demandas colocadas pela integralização da atenção à saúde, cujos limites da atuação e da intervenção são amplamente observados pela literatura da própria área, demandando novas reformulações metodológicas que incluam, em seu horizonte, perspectivas inter ou multidisciplinares ${ }^{2}$.

\section{Notas}

1. Referência ao trabalho do pesquisador Arthur Kleinman, que se tornou paradigmático dos estudos na área de antropologia médica norte-americana: Patients and Healers in the Context of Culture- an exploration of the borderland between anthropology, medicine and psychiatry, Berkeley: University of California Press, 1980.

2. Para maiores discussões sobre o tema, recomenda-se também a leitura de duas coletâneas publicadas no Brasil: Rouquayrol, M. Z. e Almeida Filho, N. (Orgs.) Epidemiologia e Saúde, Rio de Janeiro: MEDSI, 2003; e Minayo, M. C. S. e Coimbra Jr., C. E. A (Orgs.). Críticas e Atuantes: ciências sociais e humanas em saúde na América Latina, Rio de Janeiro: Fiocruz, 2005. 